\title{
Impact of Subtotal Parathyroidectomy on Clinical Parameters and Quality of Life in Hemodialysis Patients with Secondary Hyperparathyroidism
}

\author{
Mohamed Mimi Abd Elgawwad El-kholey ${ }^{1}$, Ghada El-said Ibrahim² ${ }^{2}$ Osama Ibrahim Elshahat ${ }^{1}$, Ghada El-Kannishy ${ }^{2}$ \\ ${ }^{1}$ Nephrology Unit, Mansoura New General Hospital; ${ }^{2}$ Mansoura Nephrology and Dialysis Unit (MNDU), Mansoura Faculty of \\ Medicine, Mansoura, Egypt
}

Background: Impairment of quality of life (QOL) is a key clinical characteristic of patients with end-stage renal disease (ESRD), and can be especially severe in the presence of secondary hyperparathyroidism (SHPT). Despite the proven success of parathyroidectomy (PTX) in controlling biochemical parameters in patients with severe SHPT, evidence is lacking regarding the effects of PTX on various clinical outcomes, including QOL.

Methods: Twenty ESRD patients on maintenance hemodialysis with SHPT who underwent subtotal PTX were included in an observational longitudinal study. All studied patients underwent history-taking, clinical examinations, and laboratory investigations, including a complete blood count and measurements of serum calcium, phosphorus, magnesium, parathyroid hormone (PTH), and albumin levels preoperatively and at 3 months postoperatively. QOL was assessed before surgery and at 3 months after surgery using the Kidney Disease Quality of Life 36-Item Short-Form instrument.

Results: After PTX, significant decreases in serum PTH and phosphorus levels were observed, as well as a significant increase in serum magnesium levels. Significant weight gain and improvements of QOL were also detected postoperatively.

Conclusion: Subtotal PTX seems to be an efficient alternative to medical management in uncontrolled cases of SHPT, as it is capable of controlling the biochemical derangements that occur in hyperparathyroidism. Furthermore, PTX had a beneficial effect on clinical outcomes, as shown by weight gain and improvements in all QOL scales.

Keywords: Quality of life; Renal dialysis; Hyperparathyroidism, secondary; Parathyroidectomy

\section{INTRODUCTION}

Secondary hyperparathyroidism (SHPT), which is a common problem in patients with end-stage renal disease (ESRD), is a major contributor to disability and functional decline in hemodialysis (HD) patients. SHPT has been found to be related to bone pain, fracture incidence, muscle function, cardiovascular disease, sexual function, haematopoiesis, immune function, pruritus, and calciphylaxis, all of which may influence the length and quality of life (QOL) [1]. Control of parathyroid function is one of the main targets of the management of mineral and bone disorders in chronic kidney disease (CKD) [2].

The Kidney Disease: Improving Global Outcomes international guideline group recommends that patients with stages $3-5$
Received: 13 June 2019, Revised: 30 July 2019, Accepted: 24 September 2019

Corresponding author: Ghada El-Kannishy

Mansoura Nephrology and Dialysis Unit (MNDU), Mansoura Faculty of Medicine, 60 Elgomhoria Street, Mansoura, Egypt

Tel: +20-1063388374, Fax: +20-502202772, E-mail: ghadakan@mans.edu.eg
Copyright $(2019$ Korean Endocrine Society

This is an Open Access article distributed under the terms of the Creative Commons Attribution Non-Commercial License (http://creativecommons.org/ licenses/by-nc/4.0/) which permits unrestricted non-commercial use, distribution, and reproduction in any medium, provided the original work is properly cited. 
CKD with severe hyperparathyroidism who do not respond to medical therapy should undergo parathyroidectomy (PTX) [3]. This procedure exerts an important beneficial effect by reducing the toxic effects of parathyroid hormone (PTH). Studies have primarily focused on the benefits of PTX by assessing postoperative improvements in laboratory parameters [4]. Recently, ValenteDa-Silva et al. [5] observed that improvements in survival in HD patients 1 year after PTX were associated with gains in weight and bone cell mass and improvements in health-related QOL. We hypothesized that the effects of PTX on improving QOL, clinical parameters, and biochemical parameters occur even earlier.

The aim of the present study was to evaluate the effect of PTX on clinical and biochemical parameters, as well as on QOL, in patients on maintenance HD at 3 months after PTX. Predictors of the beneficial effects of PTX were investigated.

\section{METHODS}

This prospective cohort study included 20 patients with SHPT on maintenance HD hospitalized for PTX at the nephrology department of Mansoura New General Hospital between January 2017 and January 2018. All procedures performed in the study were in accordance with the Mansoura Faculty of Medicine Institutional Research Board (MS/17.01.71) and with the 1964 Helsinki Declaration and its later amendments or comparable ethical standards. Informed consent was obtained from all individual participants included in the study. The indications for PTX were either: serum PTH levels $\geq 10$ times the upper normal limit or a lack of response to oral medications, persistent hypercalcemia despite interruption of calcium and calcitriol, a calcium-phosphorus product $>70 \mathrm{mg}^{2} / \mathrm{dL}^{2}$, pathological fractures, bone deformities, ectopic calcification, disabling arthritis, intractable pruritus, or the presence of a brown tumour. The exclusion criteria included previous hospitalization within 1 month, active inflammation or infections, malignancy, and treatment with steroids and/or immunosuppressive agents. Written informed consent was obtained from all participants. The patients underwent history-taking, clinical examinations, and evaluations of laboratory parameters (complete blood count and serum calcium, phosphorus, magnesium, PTH, and albumin levels) preoperatively and at 3 months postoperatively. Clinical evaluations were carried out before surgery and at 3 months after surgery, with an emphasis on vital signs and body mass in$\operatorname{dex}(\mathrm{BMI})$.

QOL was evaluated with the Kidney Disease Quality of Life 36-Item Short-Form (KDQOL-36) scale [6]. Patients were in- terviewed to assess their QOL before the operation and at 3 months postoperatively. The questionnaire consisted of 36 questions, divided into five sections: symptoms/problem list (12 questions), effects of kidney disease (eight questions), burden of kidney disease (four questions), the short form-12 (SF-12) physical composite score, and the SF-12 mental composite score. Patients' answers were documented in an Excel spreadsheet. The score was then calculated according to the recommendations of the KDQOL-36 Scoring Program. Each component was separately evaluated as the average of a predefined sum of questions, ranging from 0 to 3 or from 0 to 5, according to the number of possible answers. Each answer was converted to 0 to 100 , with higher scores representing better QOL.

To evaluate the impact of PTX on QOL in HD patients, the index of change of QOL for each patient was calculated for each of the five scales of QOL as follows: index of change of QOL= (postoperative score-preoperative score)/preoperative score.

\section{Preparations for surgery}

All patients were hospitalized 1 week before surgery. They were maintained on calcium tablets (500 mg three times daily) and alfacalcidol ( $1 \mu \mathrm{g}$ daily). The patients had HD sessions for 4 hours without heparin 6 to 12 hours preoperatively, and blood samples were then collected for the analysis of the laboratory parameters. A central venous catheter was introduced for intravenous calcium infusion during surgery (two ampoules of $100 \%$ calcium gluconate and $200 \mathrm{~mL}$ of sodium chloride $[0.9 \%$ $\mathrm{NaCl}]$ ).

\section{Postoperative follow-up}

After surgery patients were maintained on alfacalcidol $(1 \mu \mathrm{g}$ daily), oral calcium tablets (400 mg; two tabs three times daily, in a combination containing magnesium hydroxide, zinc, and vitamin D), and intravenous calcium infusions (10 ampoules of calcium gluconate $+100 \mathrm{~mL}$ of $0.9 \% \mathrm{NaCl}$ at a rate $40 \mathrm{of} \mathrm{mL} / \mathrm{hr}$, which was reduced to 30,20 , and $10 \mathrm{~mL} / \mathrm{hr}$ on the basis of serum calcium levels measured every 12 hours). After discharge, patients were continued on calcium tablets (500 mg, two tabs, three times daily) and alfacalcidol ( $1 \mu \mathrm{g}$ daily) for approximately 3 months, guided by weekly measurements of calcium levels.

\section{Statistical analysis}

Statistical analysis was conducted using SPSS version 22.0 (IBM Corp., Armonk, NY, USA). Mean values were compared using the paired-sample $t$ test, independent-sample $t$ test, chisquare test, Fisher exact test, and analysis of variance as appro- 
priate. The results are presented as mean \pm standard deviation or median and range. Differences were considered significant when the $P$ value was $\leq 0.05$. Correlations between the index of change of QOL (as the dependent variable) and preoperative demographic and laboratory data were calculated. Regression analysis was conducted between the index of change of QOL (as the dependent variable) and preoperative demographic and laboratory data.

\section{RESULTS}

Twenty ESRD patients on maintenance HD were included in this study (13 males and seven females). The mean age of the patients was $47.2 \pm 11.7$ years, their mean HD duration was $4.2 \pm 2.42$ years, and their mean BMI was $19.6 \pm 2.3 \mathrm{~kg} / \mathrm{m}^{2}$. Seven patients had diabetes, four had ischemic heart disease, and 10 had hypertension (Table 1).

Nineteen patients underwent subtotal PTX (95\%), and only one patient underwent an operation for the second time (i.e., a redo procedure). The pathology reports of all patients (100\%) revealed hyperplasia of the tissue of the parathyroid gland. Itching was a universal symptom, as it occurred in all patients. Furthermore, 17 patients $(85 \%)$ had generalized bone ache, and three patients $(15 \%)$ had a pathological fracture as the main presentation (data not included).

The mean preoperative serum levels of PTH, calcium, phosphorus, and magnesium were 2,214.55 $\pm 468.07 \mathrm{pg} / \mathrm{mL}, 8.70 \pm$ $0.51 \mathrm{mg} / \mathrm{dL}, 7.82 \pm 1.50 \mathrm{mg} / \mathrm{dL}$, and $1.99 \pm 0.15 \mathrm{mg} / \mathrm{dL}$, respectively. After HD, the mean preoperative levels of serum albumin, haemoglobin, and urea were $3.98 \pm 0.33 \mathrm{~g} / \mathrm{dL}, 9.40 \pm 0.89 \mathrm{~g} / \mathrm{dL}$, and $70.82 \pm 1.50 \mathrm{mg} / \mathrm{dL}$, respectively.

After PTX, there was a significant increase in BMI $(P<0.001)$, as well as significant decreases in PTH and phosphorus levels

Table 1. Preoperative Demographic and Clinical Data of the Study Participants $(n=20)$

\begin{tabular}{lc}
\hline Parameter & Value \\
\hline Male, sex & $13(65)$ \\
Age, yr & $47.2 \pm 11.7$ \\
Duration of hemodialysis, yr & $4.2 \pm 2.42$ \\
Body mass index, $\mathrm{kg} / \mathrm{m}^{2}$ & $19.6 \pm 2.3$ \\
Diabetes mellitus & $7(35)$ \\
Ischemic heart disease & $4(20)$ \\
Systemic hypertension & $10(50)$ \\
\hline Values are expressed as number $(\%)$ or mean \pm standard deviation.
\end{tabular}

(preoperative: $2,214.55 \pm 468.07 \mathrm{pg} / \mathrm{mL}$ vs. postoperative: $41.74 \pm$ $22.65 \mathrm{pg} / \mathrm{mL}$; and preoperative: $7.82 \pm 1.50 \mathrm{mg} / \mathrm{dL}$ vs. postoperative: $4.96 \pm 0.80 \mathrm{mg} / \mathrm{dL}$, respectively; $P<0.001$ ) and a significant increase in magnesium levels (preoperative: $1.99 \pm 0.15 \mathrm{mg} / \mathrm{dL}$ vs. postoperative: $2.04 \pm 0.13 \mathrm{mg} / \mathrm{dL}$, respectively; $P=0.041$ ). The changes in haemoglobin levels, white blood cell count, platelets, calcium levels, and albumin levels were non-significant (Table 2).

The preoperative scores for all the composite QOL scales were low. Three months after PTX, there was a significant increase in all scores (symptom/problem list, effects of kidney disease, and burden of kidney disease: $P<0.001, P<0.008$, and $P<0.001$, respectively). There was also a significant increase in the SF-12 physical health composite score $(P<0.012)$ and the SF-12 mental health composite score $(P<0.023)$ (Table 3$)$.

Regarding improvements in QOL, as quantified through the index of change, a significant negative correlation was found between the index of change of the symptoms/problem list scale and preoperative weight (Table 4).

In the regression analysis, the index of change of QOL was included as the dependent variable, while the independent variables were age, weight, duration of $\mathrm{HD}$, and preoperative serum calcium, PTH, albumin, phosphorus, magnesium, and hemoglobulin levels. Preoperative haemoglobin, PTH, and magnesium levels were found to be the only predictors of change (improvement) in the physical activity scale of QOL after PTX in the investigated group of HD patients (Table 5).

Table 2. Comparison of BMI and Laboratory Parameters (Preoperative and at 3 Months Postoperatively)

\begin{tabular}{lccc}
\hline Variable & Preoperative & $\begin{array}{c}3 \text { mo } \\
\text { postoperatively }\end{array}$ & P value \\
\hline BMI, kg/m & $19.6 \pm 2.3$ & $20.4 \pm 2.7$ & $<0.001^{\mathrm{a}}$ \\
PTH, $\mathrm{pg} / \mathrm{mL}$ & $2,214.55 \pm 468.07$ & $41.74 \pm 22.65$ & $<0.001^{\mathrm{a}}$ \\
Calcium, mg/dL & $8.70 \pm 0.51$ & $8.79 \pm 0.54$ & 0.731 \\
Phosphorus, mg/dL & $7.82 \pm 1.50$ & $4.96 \pm 0.80$ & $<0.001^{\mathrm{a}}$ \\
Magnesium, $\mathrm{mg} / \mathrm{dL}$ & $1.99 \pm 0.15$ & $2.04 \pm 0.13$ & $0.041^{\mathrm{a}}$ \\
Albumin, g/dL & $3.98 \pm 0.33$ & $3.99 \pm 0.30$ & 0.418 \\
Haemoglobin, g/dL & $9.40 \pm 0.89$ & $9.63 \pm 0.49$ & 0.410 \\
WBCs, $\times 10^{3} / \mathrm{mm}^{3}$ & $6.18 \pm 1.60$ & $6.03 \pm 1.30$ & 0.735 \\
Platelets, $\times 10^{3} / \mathrm{mm}^{3}$ & $249.70 \pm 60.38$ & $256.95 \pm 62.74$ & 0.510 \\
\hline
\end{tabular}

Values are expressed as mean \pm standard deviation.

BMI, body mass index; PTH, parathyroid hormone; WBC, white blood cell.

a Significant at $P \leq 0.05$. 
Table 3. Comparison of Quality of Life Scores (Pre-PTX and at 3 Months Post-PTX)

\begin{tabular}{lrrr}
\hline Variable & Pre-PTX & Post-PTX & $P$ value \\
\hline Symptom/problem list & $58.23 \pm 7.63$ & $77.66 \pm 6.22$ & $<0.001^{\mathrm{a}}$ \\
Effects of kidney diseases & $28.44 \pm 3.78$ & $45.72 \pm 7.16$ & $<0.008^{\mathrm{a}}$ \\
Burden of kidney diseases & $4.69 \pm 7.55$ & $39.8 \pm 20.01$ & $<0.001^{\mathrm{a}}$ \\
SF-12 physical health composite & $25.65 \pm 4.53$ & $44.32 \pm 4.98$ & $<0.012^{\mathrm{a}}$ \\
SF-12 mental health composite & $31.88 \pm 1.72$ & $49.83 \pm 3.32$ & $<0.023^{\mathrm{a}}$ \\
\hline
\end{tabular}

Values are expressed as mean \pm standard deviation.

PTX, parathyroidectomy; SF, short form.

${ }^{\text {a }}$ Significant at $P \leq 0.05$.

Table 4. Correlations between the Index of Change of Quality of Life and Preoperative Demographic and Laboratory Parameters

\begin{tabular}{|c|c|c|c|c|c|c|c|c|c|c|}
\hline \multirow{2}{*}{ Variable } & \multicolumn{2}{|c|}{ Symptoms } & \multicolumn{2}{|c|}{ Effects } & \multicolumn{2}{|c|}{ Burden } & \multicolumn{2}{|c|}{ Physical } & \multicolumn{2}{|c|}{ Mental } \\
\hline & $r$ & $P$ value & $r$ & $P$ value & $r$ & $P$ value & $r$ & $P$ value & $r$ & $P$ value \\
\hline Age & -0.235 & 0.332 & -0.155 & 0.527 & 0.034 & 0.889 & -0.106 & 0.666 & 0.141 & 0.566 \\
\hline Weight & -0.589 & 0.008 & -0.032 & 0.898 & -0.225 & 0.354 & -0.204 & 0.403 & 0.142 & 0.561 \\
\hline Duration of hemodialysis & 0.197 & 0.420 & -0.029 & 0.906 & -0.265 & 0.273 & 0.001 & 0.998 & -0.226 & 0.352 \\
\hline Preoperative serum calcium levels & 0.149 & 0.542 & 0.006 & 0.981 & -0.416 & 0.077 & 0.087 & 0.724 & 0.019 & 0.937 \\
\hline Preoperative serum PTH levels & -0.349 & 0.143 & 0.321 & 0.180 & 0.144 & 0.557 & -0.165 & 0.499 & 0.258 & 0.286 \\
\hline Preoperative serum albumin levels & -0.425 & 0.070 & 0.060 & 0.809 & 0.254 & 0.294 & -0.207 & 0.396 & 0.212 & 0.383 \\
\hline Preoperative haemoglobin & -0.026 & 0.914 & 0.272 & 0.259 & -0.036 & 0.885 & -0.231 & 0.342 & 0.273 & 0.259 \\
\hline Preoperative phosphorus levels & -0.323 & 0.177 & 0.437 & 0.061 & -0.086 & 0.727 & -0.311 & 0.195 & 0.314 & 0.190 \\
\hline Preoperative magnesium levels & 0.040 & 0.871 & 0.461 & 0.05 & -0.162 & 0.508 & -0.191 & 0.433 & 0.122 & 0.618 \\
\hline
\end{tabular}

Table 5. Regression Analysis with Index of Change of Quality of Life Scores as the Dependent Variable

\begin{tabular}{|c|c|c|c|c|c|c|c|c|c|c|}
\hline \multirow{2}{*}{ Variable } & \multicolumn{2}{|c|}{ Symptoms } & \multicolumn{2}{|c|}{ Effects } & \multicolumn{2}{|c|}{ Burden } & \multicolumn{2}{|c|}{ Physical } & \multicolumn{2}{|c|}{ Mental } \\
\hline & $r$ & $P$ value & $r$ & $P$ value & $r$ & $P$ value & $r$ & $P$ value & $r$ & $P$ value \\
\hline Age & 0.644 & 0.586 & 0.761 & 0.526 & 1.373 & 0.304 & 3.364 & 0.078 & 0.102 & 0.928 \\
\hline Weight & 0.684 & 0.565 & 0.180 & 0.874 & 1.998 & 0.184 & 2.309 & 0.147 & 0.773 & 0.520 \\
\hline Duration of hemodialysis & 0.634 & 0.591 & 0.071 & 0.950 & 1.655 & 0.240 & 3.154 & 0.088 & 0.866 & 0.478 \\
\hline Preoperative serum calcium levels & 0.316 & 0.782 & 1.091 & 0.389 & 1.620 & 0.247 & 3.219 & 0.084 & 0.145 & 0.898 \\
\hline Preoperative serum PTH levels & 0.076 & 0.946 & 1.913 & 0.196 & 0.138 & 0.903 & 8.892 & 0.012 & 0.780 & 0.517 \\
\hline Preoperative serum albumin levels & 1.029 & 0.412 & 1.914 & 0.196 & 2.998 & 0.096 & 0.613 & 0.602 & 0.570 & 0.626 \\
\hline Preoperative haemoglobin & 0.964 & 0.437 & 0.464 & 0.688 & 2.783 & 0.108 & 4.863 & 0.040 & 0.070 & 0.951 \\
\hline Preoperative phosphorus levels & 1.111 & 0.382 & 1.653 & 0.240 & 1.522 & 0.267 & 1.114 & 0.381 & 0.183 & 0.871 \\
\hline Preoperative magnesium levels & 0.455 & 0.694 & 1.067 & 0.398 & 3.173 & 0.087 & 5.382 & 0.033 & 0.676 & 0.569 \\
\hline
\end{tabular}

PTH, parathyroid hormone.

\section{DISCUSSION}

SHPT is a common problem in the management of ESRD. Although hyperparathyroidism can be controlled medically or sur- gically, severe hyperparathyroidism may increase the difficulty of medical control. The present study investigated the effects of PTX in HD patients with severe SHPT on QOL, clinical parameters, and biochemical parameters. Twenty ESRD patients $(65 \%$ 
males; mean age, $47.2 \pm 11.7$ years) with SHPT who were on regular HD and hospitalized for PTX.

In the current study, significant decreases in serum PTH and phosphorus levels were observed at 3 months postoperatively, but a non-significant change in calcium levels was found. These data are in agreement with a previous study conducted among 60 patients, in which the authors documented a decline of PTH serum levels from a mean preoperative level of $2,357 \mathrm{pg} / \mathrm{mL}$ (range, 819 to 4,697) to a mean postoperative serum level of $128 \mathrm{pg} / \mathrm{mL}$ (range, 1.3 to 967 ) during a mean follow-up of 36 months [7]. Peters et al. [8] found that significant decreases in serum phosphorus and intact PTH levels occurred after PTX, while there was a non-significant difference in serum calcium levels. On the contrary, in 2017, Valente-Da-Silva et al. [5] found significant decreases in PTH and phosphorus levels after total PTX with a forearm implant, in association with significant decreases in calcium levels 1 year after surgery. This documented decrease in serum calcium levels may have been related to reduced calcium intake or a longer effect of surgery [5]. It should be noted that the patients included in the current study continued to take calcium tablets and alfacalcidol, guided by calcium level measurements obtained every week.

The normal adult serum value for magnesium is 1.5 to 2.5 $\mathrm{mEq} / \mathrm{L}$ (1.7 to $2.4 \mathrm{mg} / \mathrm{dL}$ ). Clinical and epidemiological studies have shown low serum magnesium levels to be associated with vascular calcification and cardiovascular mortality in ESRD patients, but the pathophysiology and the role of magnesium disturbances in CKD remain unclear, making further studies necessary [9]. In dialysis patients, an inverse relationship between serum magnesium concentrations and serum PTH levels was observed [10,11]. However, Sanda et al. [12] stated that PTH could elevate serum magnesium levels by enhancing its gastrointestinal absorption and bone resorption. In 2015, Fang et al. [13] documented a case of preoperative hypermagnesemia. They suggested that magnesium liberated by PTH induced excessive osteoclastic bone resorption. PTX could stimulate hungry bone syndrome, causing a postoperative reduction of serum magnesium, which might be due to the movement of magnesium out of the extracellular space into the cells or to be deposited in the bones [13]. It was suggested that patients on dialysis are largely dependent on the magnesium concentration in the dialysate to maintain their serum magnesium levels. Katopodis et al. [14], in a 2003 study, found that mild hypermagnesemia occurred when using a dialysate magnesium concentration of $0.75 \mathrm{mmol} / \mathrm{L}$ in both peritoneal dialysis and HD patients, while the results were not as consistent when a lower dialysate concentration ( $0.5 \mathrm{and} /$ or $0.25 \mathrm{mmol} / \mathrm{L}$ ) was used. In the current study, the mean level of magnesium before surgery was within the normal range, despite the high mean PTH level. The dialysate used at our institution had a magnesium concentration of $0.5 \mathrm{mmol} / \mathrm{L}$. According to the results of the present study, there was a significant increase in serum magnesium 3 months after surgery.

PTH is considered to be a catabolic agent that plays a role in wasting syndrome and contributes in a modest manner to the accumulation of nitrogenous waste products in the blood [15]. Three months postoperatively, clinical parameters were re-evaluated in the study population, and significant weight gain was noticed (preoperative BMI: $19.6 \pm 2.3 \mathrm{~kg} / \mathrm{m}^{2}$ vs. postoperative BMI: $\left.20.4 \pm 2.7 \mathrm{~kg} / \mathrm{m}^{2}, P<0.001\right)$. In agreement with these results, Valente-Da-Silva et al. [5] reported that PTX with forearm implantation performed in ESRD patients on regular HD was associated with weight gain. An increase in weight after PTX could be explained by the fall of serum phosphorus levels, which have a toxic cellular effect that causes the total body mass to decrease [16]. However, in 2006, Peters et al. [8] observed a negative correlation between intact PTH levels and total body fat before PTX. They concluded that weight and bioelectrical impedance parameters were unaffected by surgery.

Serum albumin levels did not show any significant changes at 3 months post-PTX in the studied population. Valente-Da-Silva et al. [5] identified a non-significant decrease in the proportion of subjects with low albumin levels, from $33 \%$ before surgery to $26 \%$ at 1 year after surgery. In contrast, in 2006 , Peters et al. [17] revealed that serum albumin levels significantly increased 6 months post-PTX in patients with SHPT on HD.

Elevated PTH levels are associated with bone marrow fibrosis and systemic inflammation. PTX causes a rapid decrease in intact PTH levels, which can positively affect renal anaemia and nutritional status. Some previous reports documented a significant increase in haemoglobin levels after PTX $[5,18]$. Data from the present study documented an increase in haemoglobin 3 months after the operation; however, the difference was statistically non-significant.

According to our results, poor QOL (including all composite scales of QOL) was evident before PTX. This is in agreement with Malindretos et al. [19], who studied health-related QOL using the KDQOL-36 questionnaire in ESRD patients on regular HD treatment at six dialysis clinics in Greece, and observed poor QOL before surgery that was not directly related to duration of the disease.

In the current study, PTX yielded a significant improvement in all physical and mental items of the KDQOL-36. Significant 
improvements in all five scales (symptom/problem list, effects of kidney disease, burden of kidney disease, physical health composite score, and mental health composite score) were documented. This effect may have taken place because the toxic properties of PTH diminished. These results are in accordance with those of Cheng et al. [20], who performed a prospective study that included 49 patients who underwent PTX for SHPT. In that study, participants completed the SF-36 questionnaires before PTX and at 12 months postoperatively. The authors documented that PTX had a significant beneficial effect on all physical and mental items, and they concluded that PTX is indicated as early as possible in CKD patients with SHPT and poor QOL [20].

In the patients recruited in the present study, QOL improvements were not correlated with age, duration of $\mathrm{HD}$, or preoperative levels of calcium, phosphorus, and PTH. However, there was a significant negative correlation between improvement in the symptoms scale (expressed as its index of change) and preoperative weight. As a possible explanation for the latter relationship, those patients may have experienced particularly severe toxic effects of high PTH levels, resulting in more weight loss and greater improvements after the reversal of their PTH levels.

Bratucu et al. [21] analysed 85 consecutive unselected patients who underwent total PTX for SHPT, and documented that there was no correlation between preoperative PTH or calcium levels and clinical symptoms. Their study also revealed that PTX clearly led to improvements in QOL in SHPT patients, with a durable effect at 6 months [21].

To the best of our knowledge, no previous studies have investigated predictors of improvement in QOL post-PTX. The present study demonstrated that preoperative levels of PTH, haemoglobin, and magnesium were the only predictors of improvement in the physical activity scale of QOL after PTX in HD patients.

In conclusion, PTX is an efficient alternative for the management of SHPT in selected patients, as it is capable of controlling the biochemical derangements that occur in hyperparathyroidism. PTX also had a beneficial effect on clinical outcomes, as shown by improvements in QOL and weight gain. However, this study was observational and non-controlled, making it difficult to fully establish the possible association between PTX and QOL in this series of patients with SHPT on HD. Prospective studies with a larger sample size are needed to provide evidence regarding this possibility and to investigate the causes of the observed changes in QOL. Comparing the effects of differ- ent strategies of SHPT treatment in HD patients on QOL appears to be a promising topic for further investigations.

\section{CONFLICTS OF INTEREST}

No potential conflict of interest relevant to this article was reported.

\section{AUTHOR CONTRIBUTIONS}

Conception or design: G.E.K. Acquisition, analysis, or interpretation of data: M.M.A.E.E. Drafting the work or revising: O.I.E. Final approval of the manuscript: G.E.I.

\section{ORCID}

Mohamed Mimi Abd Elgawwad El-kholey https://orcid.org/ 0000-0002-8574-7773

Ghada El-Kannishy https://orcid.org/0000-0003-2016-5235

\section{REFERENCES}

1. Rodriguez M, Lorenzo V. Parathyroid hormone, a uremic toxin. Semin Dial 2009;22:363-8.

2. de Brito Galvao JF, Nagode LA, Schenck PA, Chew DJ. Calcitriol, calcidiol, parathyroid hormone, and fibroblast growth factor-23 interactions in chronic kidney disease. J Vet Emerg Crit Care (San Antonio) 2013;23:134-62.

3. Kidney Disease: Improving Global Outcomes (KDIGO) CKD-MBD Work Group. KDIGO clinical practice guideline for the diagnosis, evaluation, prevention, and treatment of Chronic Kidney Disease-Mineral and Bone Disorder (CKD-MBD). Kidney Int Suppl 2009;130:S1-130.

4. Trombetti A, Stoermann C, Robert JH, Herrmann FR, Pennisi $\mathrm{P}$, Martin PY, et al. Survival after parathyroidectomy in patients with end-stage renal disease and severe hyperparathyroidism. World J Surg 2007;31:1014-21.

5. Valente-Da-Silva HG, Maya MCA, Moreira AS. Parathyroidectomy in chronic kidney disease: effects on weight gain and on quality of life improvement. Rev Col Bras Cir 2017; 44:263-9.

6. RAND Health Care. Kidney Disease Quality of Life Instrument (KDQOL) [Internet]. Santa Monica (CA): RAND Corp., 2019 [cited 2019 Oct 24]. Available from: https://www.rand. org/health-care/surveys_tools/kdqol.html.

7. Ma TL, Hung PH, Jong IC, Hiao CY, Hsu YH, Chiang PC, 
et al. Parathyroidectomy is associated with reduced mortality in hemodialysis patients with secondary hyperparathyroidism. Biomed Res Int 2015;2015:639587.

8. Peters BS, Jorgetti V, Martini LA. Body composition changes in haemodialysis patients with secondary hyperparathyroidism after parathyroidectomy measured by conventional and vector bioimpedance analysis. Br J Nutr 2006;95:353-7.

9. Dousdampanis P, Trigka K, Fourtounas C. Hypomagnesemia, chronic kidney disease and cardiovascular mortality: pronounced association but unproven causation. Hemodial Int 2014;18:730-9.

10. Brown EM, MacLeod RJ. Extracellular calcium sensing and extracellular calcium signaling. Physiol Rev 2001;81:239-97.

11. Ohya M, Negi S, Sakaguchi T, Koiwa F, Ando R, Komatsu Y, et al. Significance of serum magnesium as an independent correlative factor on the parathyroid hormone level in uremic patients. J Clin Endocrinol Metab 2014;99:3873-8.

12. Sanda S, Schlingmann KP, Newfield RS. Autosomal dominant hypoparathyroidism with severe hypomagnesemia and hypocalcemia, successfully treated with recombinant PTH and continuous subcutaneous magnesium infusion. J Pediatr Endocrinol Metab 2008;21:385-91.

13. Fang L, Tang B, Hou D, Meng M, Xiong M, Yang J. Effect of parathyroid hormone on serum magnesium levels: the neglected relationship in hemodialysis patients with secondary hyperparathyroidism. Ren Fail 2016;38:50-6.

14. Katopodis KP, Koliousi EL, Andrikos EK, Pappas MV, Elisaf MS, Siamopoulos KC. Magnesium homeostasis in patients undergoing continuous ambulatory peritoneal dialysis: role of the dialysate magnesium concentration. Artif Organs 2003;27:853-7.

15. Massry SG. The toxic effects of parathyroid hormone in uremia. Semin Nephrol 1983:3:306-28.

16. Ohnishi M, Razzaque MS. Dietary and genetic evidence for phosphate toxicity accelerating mammalian aging. FASEB J 2010;24:3562-71.

17. Peters BS, Jorgetti V, Martini LA. Body composition changes in haemodialysis patients with secondary hyperparathyroidism after parathyroidectomy measured by conventional and vector bioimpedance analysis. Br J Nutr 2006;95:353-7.

18. Chow TL, Chan TT, Ho YW, Lam SH. Improvement of anemia after parathyroidectomy in Chinese patients with renal failure undergoing long-term dialysis. Arch Surg 2007;142: 644-8.

19. Malindretos P, Sarafidis P, Lazaridis A, Nikolaidis P. A study of the association of higher parathormone levels with healthrelated quality of life in hemodialysis patients. Clin Nephrol 2012;77:196-203.

20. Cheng SP, Lee JJ, Liu TP, Yang TL, Chen HH, Wu CJ, et al. Parathyroidectomy improves symptomatology and quality of life in patients with secondary hyperparathyroidism. Surgery 2014;155:320-8.

21. Bratucu MN, Garofil ND, Radu PA, Paic V, Zurzu M, Goleanu V, et al. Surgical attitude in patients with secondary hyperparathyroidism undergoing dialysis. Chirurgia (Bucur) 2015; 110:418-24. 